\title{
Fourth ventricle primitive choroid plexus papilloma with divergent differentiation potentials: A case report
}

\author{
Rosalba Vega-Orozcoํㅜ, Martha Lilia Tena-Suck ${ }^{2}$, Daniel Rembao Bojórquez ${ }^{1}$, Miguel Ángel \\ Collado-Ortiz ${ }^{2}$
}

1. Department of Neuropathology, National Institute of Neurology and Neurosurgery, México City, Mexico. 2. Neurological center, Department of Clinical Neurophisiology. The American British Cowdray Hospital, Mexico City, Mexico.

Correspondence: Martha Lilia Tena-Suck, M.D. Address: Department of Neuropathology, National I nstitute of Neurology and Neurosurgery "Manuel Velasco Suárez", Av Insurgentes Sur 3877 Col. La Joya, Delegación Tlalpan CP 14269, Mexico City, Mexico. E-mail: mltenasuck@gmail.com

Received: February 27, 2015

DOI : $10.5430 /$ crcp.v2n4p1
Accepted: April 9, 2015

URL: http://dx.doi.org/10.5430/crcp.v2n4p1
Online Published: April 16, 2015

\begin{abstract}
s
Choroid plexus tumors are rare tumors, although choroid plexus tumours account for $0.3 \%-0.6 \%$ of all brain tumors. We presented a rare case in four ventricle tumor, a primitive choroid plexus Papilloma with divergent differentiation potentials. In 43-year-old male, he started with postural vertigo, occipital cephalea, temporary diplopia and right lateropulsion from two years ago. MRI showed hyperintense tumor in the fourth ventricle with signs of cerebral edema and hemangioblastoma was proposed. Microscopically the tumor was composed of two histological components; one consists of blood vessels of different sizes and capillaries type a rich anastomosing network with these stromal cells are epithelioid-looking, broad with eosinophilic cytoplasm, and occasionally were vacuolated or foamy cytoplasm. The second one composed of epithelial cells forming papillae structures of varying sizes and shape with endothelial hyperplasia and some papillae presenting reached glomeruli-like structures, with ependymoma and haemangioblastoma appearance. The case presented may constitute a new neoplastic entity within the recently described spectrum of central nervous system tumors with ependymal features. Just two cases of this rare combination of different histological patterns have been reported in choroid with displacement and maldifferentiation of primitive, multipotent neuroepithelial cells.
\end{abstract}

\section{Key words}

Choroid plexus papilloma, Choroid plexus tumors, Ependymoma, Haemangioblastoma, Divergent differentiation

\section{I ntroduction}

Choroid plexus tumors (CPT) are rare neoplasms derived from choroid plexus epithelium ${ }^{[1]}$. CPT account for $0.3 \%-0.6 \%$ of all brain tumors and they represent $2 \%-4 \%$ of those that occur in children under 15 years and $10 \%-20 \%$ in the first year of the life ${ }^{[1]}$. The $80 \%$ of those tumours located in lateral ventricle present in children, fourth ventricle tumors are evenly distributed in all age groups ${ }^{[2,3]}$.

CPP, ependymoma and haemangioblastoma should be included as a differential diagnosis in presenting with tumors in the fourth ventricle ${ }^{[3,4]}$. 
Haemangioblastoma (HB), a rare benign tumor consists of two main components: capillaries and stromal cells ${ }^{[3-6]}$. Deletion analysis of the von Hippel Lindau gene locus has identified the stromal cells as neoplastic ${ }^{[1]}$, despite many efforts, the histogenesis of stromal cells is still unknown. But their origin remains enigmatic, suggested origins included glial cells, endothelial cells, arachnoids cells, embryonic choroid plexus or neuroendocrine cells derivation ${ }^{[1]}$.

We report a rare case of choroid plexus Papilloma with haemangioblastoma and ependymoma differentiation in the same tumor, in four ventricle located.

\section{Case report}

A 43-year-old male, he started with postural vertigo, occipital cephalea, temporary diplopia and right lateropulsion from two years ago. He presented left dysmetria. The TAC and the MRI showed hyperintense tumor in the fourth ventricle with signs of cerebral edema. Hemangioblastoma was proposed (see Figure 1a). The tumor was grossly totally resected. However, during the trans-operative procedure the patient bled significantly and is now in coma.

Microscopically the tumor was composed of two histological components; one consists of blood vessels of different sizes and capillaries type a rich anastomosing network (see Figure 1b), with these stromal cells are epithelioid-looking, broad with eosinophilic cytoplasm, and occasionally were vacuolated or foamy cytoplasm. The second one composed of epithelial cells forming papillae structures of varying sizes and shape (see Figure 1c), with fibro stems containing several blood vessels and clear stromal cells (see Figure 1d). On the inside of the papillae was observed proliferation of blood vessels with endothelial hyperplasia (see Figure 1e), and some papillae presenting reached glomeruli-like structures. In other areas were observed clear ciliated epithelial cells with nuclei to the periphery vascular lumen. Not atypia or mitotic figures were observed (see Figure 1f). Some papillae had ependymoma appearance. Also was observed in the intravellous stroma abundant capillaries and clear cytoplasm stromal cells, and few dystrophic calcifications (see Figure 2a). Ependymal epithelium was observed around the tumor with reactive gliosis and Rosenthal fibres (see Figure 2b), as well as infiltration of clear cells forming rosette-like structures (see Figure 2c), and single neoplastic cells in the adjacent brain parenchyma (see Figure 2d). Choroid plexus Papilloma with haemangioblastoma and ependymoma components was diagnosed.

Immunostaining was performed by the peroxidase-antiperoxidase method. The characteristics of the primaries antibodies used and the results are seen in Table 1. GFAP was positive in brain tumor boundary (see Figure 2e) and diffuse brain infiltration (see Figure 2f). Cytokeratin 8/18 was strong positive in the cells that lined the papillae and negative in vessels and in the spongy cells (see Figure 3a). Synaptophysin, CD68, factor VIII (see Figure 3b), vimentin (see Figure 3c), and S-100 were strongly positive epithelial cells and endothelial cells in the spongy cells (see Figure 3d). EVGF (see Figure 3e), EVGF-RII and HIF-1 $\alpha$ (see Figure 3f) were positive in the vessels and spongy cells, negative in epithelial cells of the papillae. NSE, synaptophysin, neurofilament, and tubulin were weakly positive in the stromal cells and focally in epithelial cells. P53, gastrin and chromogranin A were negative in both cells. The mib-1(ki-67) labelling index was 4\%.

\section{Discussion}

Few cases have been published about this rare association ${ }^{[1-3]}$. The first case of this tumor was published by Lowenthal in $1950^{[2]}$ that reported a case of Cerebellar hemangioblastoma, with meningeal hemangioblastoma, osseous metaplasia, hypertrophy of the choroid plexus and amorphous calcium overload, in the same subject. Other one was published by Dulai MS, et al. ${ }^{[2]}$ an intramedular papillary ependymoma with choroid plexus differentiation and cerebrospinal fluid dissemination to the brain. Cai CE et al. ${ }^{[7]}$, published a case a cystic mass with a mural enhancing nodule centered within the cerebellar vermis with exuberant piloid gliosis secondary to infiltration of the CPP component into the adjacent brain parenchyma. Dulai SM et al. ${ }^{[8]}$ noted a rare spinal papillary ependymoma exhibiting mixed ependymal and choroid plexus-like differentiation. Mobley B et al. ${ }^{[9]}$, showed two tumors with papillary and solid cytoarchitecture with 
monomorphous epithelioid cells arranged around vascular papillae. Immunohistochemistry revealed diffuse epithelial membrane antigen, cytokeratin, and E-cadherin reactivity. Glial fibrillary acidic protein staining was focal in case 1 and negative in case 2. Neural cell adhesion molecule showed patchy membranous reactivity. Electron microscopy showed ependymal differentiation. Iwasaki T et al. ${ }^{[10]}$, and Bollo $\mathrm{C}$ et al. ${ }^{[11]}$ reported a solid and cyst intramedullary spinal tumor. Histologic examination showed a tumor with two distinct components, characteristics of choroid plexus papilloma and an ependymoma, WHO grade II. Hayashi Y et al. ${ }^{[12]}$ reported a rare case with multiple neuroepithelial tumor of different histological cell types. In this patient, synchronous appearance of tumors, cervical ependymoma and a fourth ventricle choroid plexus papilloma with displacement and dedifferentiation of primitive, multipotent neuroepithelial cells ${ }^{[10]}$. Furthermore, Paquier B et al. ${ }^{[13]}$ suggested that suggest that chordoid glioma of the third ventricle could be better classified as chordoid ependymoma of the lamina terminalis area.
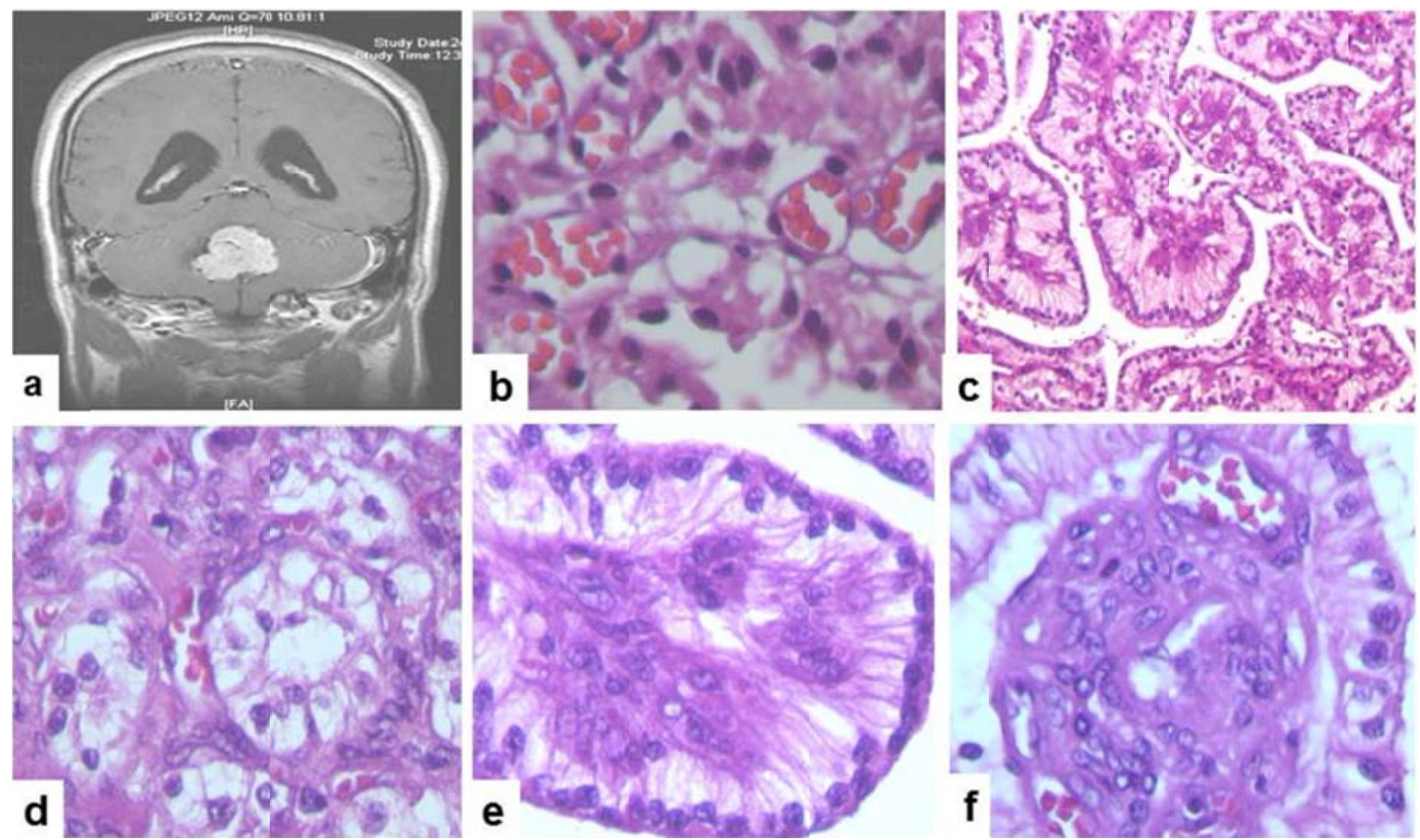

Figure 1. (a) MR Imaging representative revealed a homogeneous and isointense lesion on the T1. Coronal sections showed a large fourth ventricular enhancement mass with evidence of mild hydrocephalus. Histopathological features. (b) Tumor was composed of two histological components observed rich anastomosing network consists of blood vessels of different sizes and papillae formation. (c) Observed papillae structures composed of epithelial cells with fibro stems containing several blood vessels, in (d) observed in the intravellous stroma abundant capillaries and clear stromal cells. (e) Close up of vessels proliferation intravellous stroma and in (f) observed some papillae structure showed hyperplasic endothelial proliferation forming glomeruli like structures $(\mathrm{H} \& \mathrm{E} \times 400)$.

This rare association between choroid plexus Papilloma abundant blood vessels and haemangioblastous changes has been known as choroid plexus angioma ${ }^{[1]}$. Changes are not the issue even as it is unclear whether hyperplasia corresponds to plexus Papilloma changes or fall or if a real or merely haemangioblastoma; angiomatosis changes or corresponds to a true arteriovenous malformation in the choroid plexus, teratoma, as well as a hamartoma suggesting abnormal cell migration has been considerate ${ }^{[2]}$.

Histopathological and clinical course of rare spinal papillary ependymoma exhibiting mixed ependymal and choroid plexus-like differentiation ${ }^{[7]}$. Cytological and immunohistochemical features overlapping those of classic ependymomas and choroid plexus tumors ${ }^{[1]}$. Numerous vessels were observed in CPT or could formed by may stimulate vascular malformations, infarctions, or infections. 

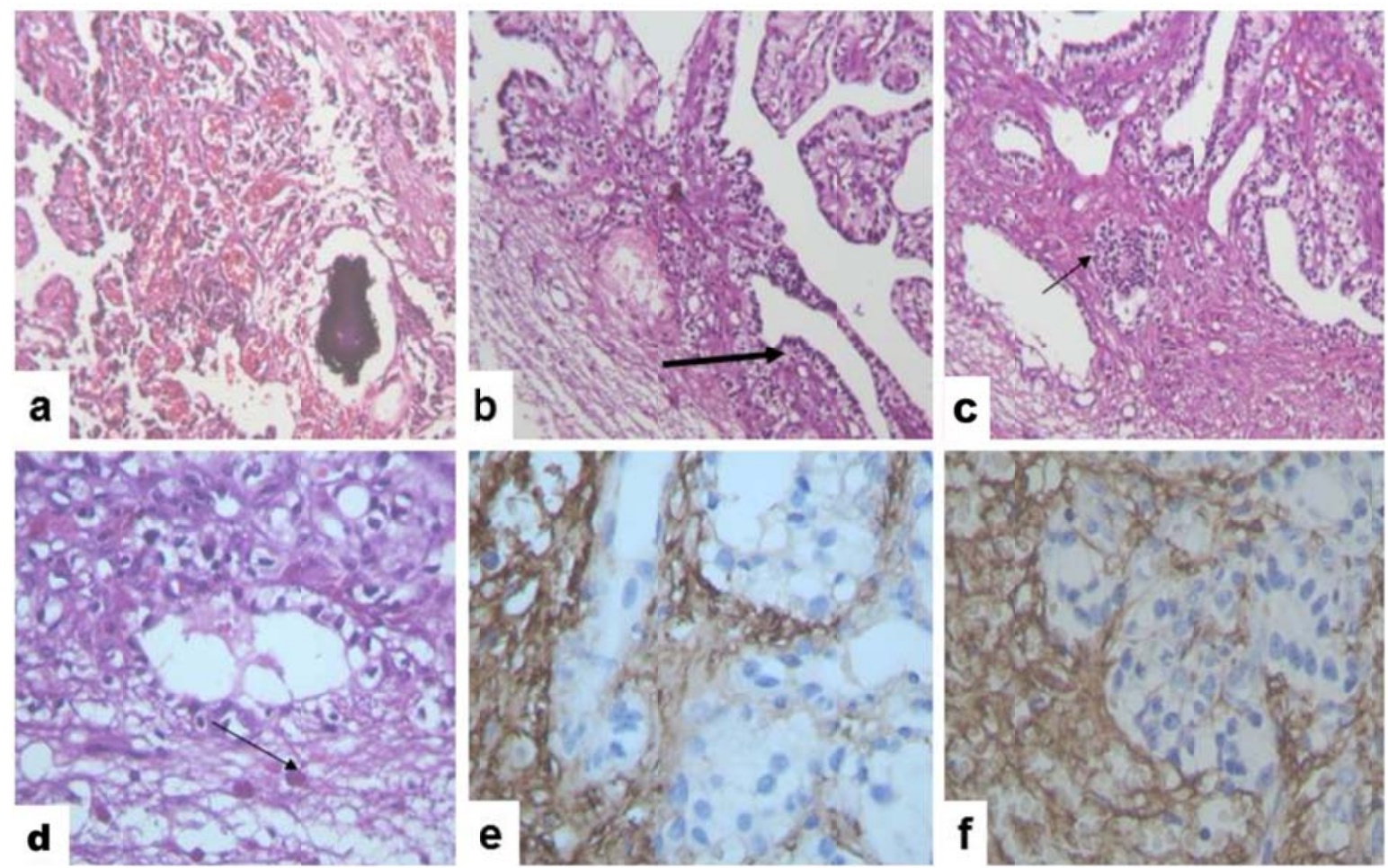

Figure 2. Histopathological features showed brain invasion. (a) Foci of dystrophic calcification were observed between papillae formation and vessels proliferation. (b) Showed the neuroepthelial fourth ventricle walls broken with foci of neoplastic cells embedded in brain parenchyma and gliosis. (c) Observed neoplastic cells into the adjacent brain parenchyma (H\&E $\times 200)$. (d) Around the tumor was observed reactive gliosis and Rosenthal fibres (The arrows showed the Rosenthal fibres) (H\&E $\times 400)$. (e and f) Observed that brain tissue was strong positive to GFAP and was negative in neoplastic a cell, that allows us to better observe the neoplastic cells infiltrating the brain parenchyma (IHQ stain original magnification $\times 400$ ).
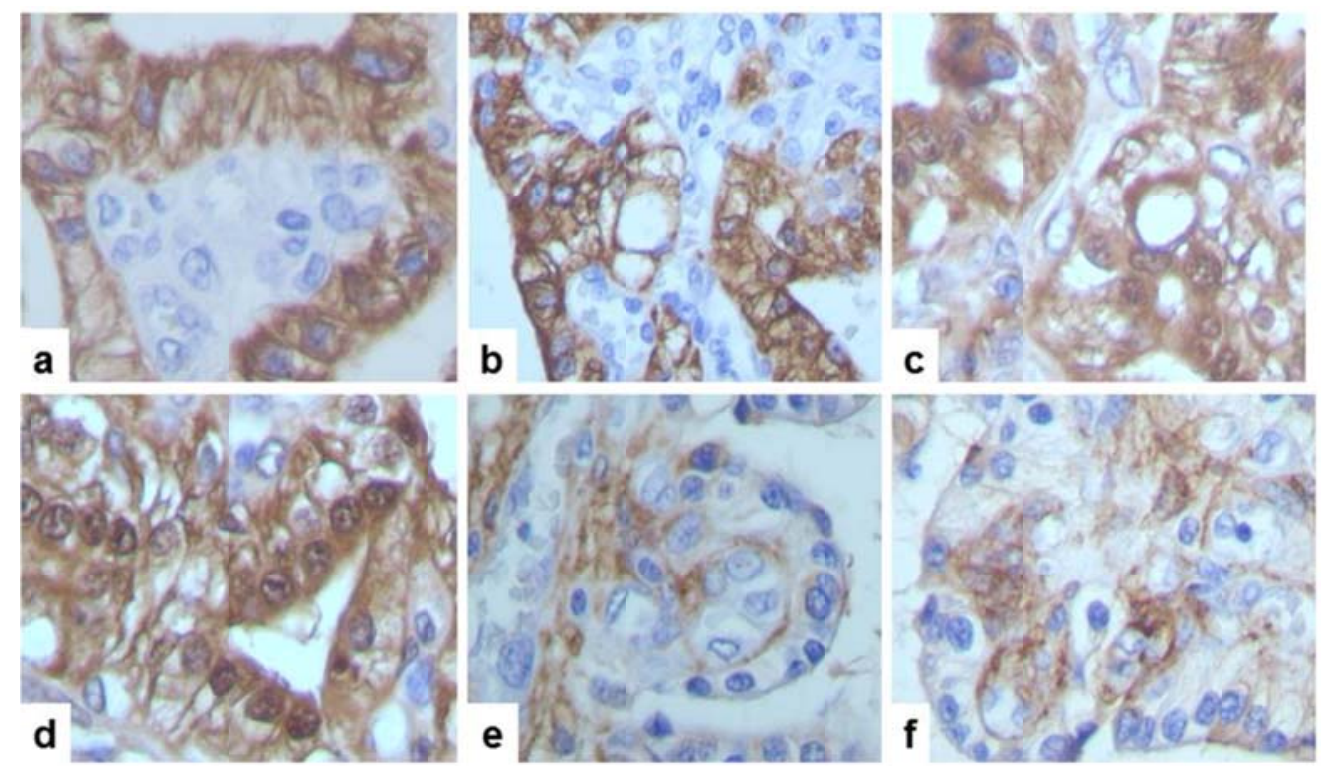

Figure 3. Immunohistochemistry. (a) Cytokeratin 18 was strong positive in the cells that lined the papillae and negative in vessels and in the spongy cells. (b) S-100, (c) Factor VIII, (d) Vimentin, (e) EVGF were strongly positive epithelial cells, endothelial cells and in the spongy cells and in (f) HIF-1 $\alpha$ positive immunoreaction in stromal cells while the epithelial cells were negative (IHQ $\times 400)$. 
Table 1. Characteristics of primary antibodies used and results

\begin{tabular}{|c|c|c|c|c|c|c|}
\hline Antibody & Clone & Source & Dilution & Epithelial & Endothelial & Stromal \\
\hline Keratin 18 & DC10 & DAKO & Prediluided & Strong & Negative & Negative \\
\hline Keratin 7 & OV-TL12/30 & DAKO & $1: 100$ & Weak & Negative & Negative \\
\hline Keratin 20 & $\mathrm{Ks} 20.8$ & DAKO & $1: 100$ & Negative & Negative & Negative \\
\hline AE1/AE3 & AE1/AE3 & DAKO & $1: 100$ & Negative & Negative & Negative \\
\hline EMA & E29 & DAKO & $1: 100$ & Negative & Negative & Negative \\
\hline GFAP & LSAB2 & DAKO & $1: 100$ & Negative & Negative & Negative \\
\hline S-100 protein & Z0311 & DAKO & $1: 100$ & Strong & Moderate & Moderate \\
\hline Actin alpha smooth muscle & 1A4 & DAKO & $1: 100$ & Negative & Negative & Negative \\
\hline Synaptophysin & Sv38 & DAKO & $1: 100$ & Strong & moderate & Moderate \\
\hline NSE & BBS/H14 & DAKO & $1: 100$ & Weak & Weak & Weak \\
\hline Neurofilament & NR4 & DAKO & $1: 100$ & Weak & Negative & Weak \\
\hline Laminin & $4 \mathrm{C} 7$ & DAKO & $1: 100$ & Negative & Negative & Negative \\
\hline P53 & DO-7 & DAKO & $1: 100$ & Negative & Negative & Negative \\
\hline Tubulin- $\alpha$ & Sc5286 & $\mathrm{SC}$ & $1: 100$ & Weak & Negative & Negative \\
\hline CD34 & QBEen10 & DAKO & $1: 100$ & Negative & Strong & Negative \\
\hline vWF & $\mathrm{F} 8 / 86$ & DAKO & $1: 100$ & Weak & Strong & Weak \\
\hline CD38 & AT1 & $\mathrm{SC}$ & $1: 100$ & Negative & Negative & Negative \\
\hline CD68 & EBM11 & $\mathrm{SC}$ & $1: 100$ & Negative & Weak & Weak \\
\hline E-Cadherin & Sc52327 & BIOCARE & Prediluided & Weak & Negative & Negative \\
\hline $\mathrm{ACE}$ & II-7 & DAKO & Prediluided & Negative & Negative & Negative \\
\hline A-feto protein & LSAB2 & DAKO & Prediluided & Negative & Negative & Negative \\
\hline Vimentin & V9 & DAKO & $1: 100$ & Moderate & Strong & Moderate \\
\hline Gastrin & LSAB2 & DAKO & $1: 100$ & Negative & Negative & Negative \\
\hline Chromogranin & DAK-A3 & DAKO & $1: 100$ & Negative & Negative & Negative \\
\hline EGF & MO886 & DAKO & $1: 100$ & Negative & Negative & Negative \\
\hline EVGF & $5 \mathrm{C} 3 . \mathrm{F} 8$ & $\mathrm{SC}$ & $1: 100$ & Negative & Moderate & Moderate \\
\hline NGFR & NGFR5 & DAKO & $1: 100$ & Weak & Moderade & Moderate \\
\hline HIF-1 $\alpha$ & HI $\alpha-67$ & $\mathrm{SC}$ & $1: 100$ & Negative & Moderate & Moderate \\
\hline
\end{tabular}

Note. Epithelial Membrane antigen (EMA), Glial Fibrillary acidic Protein (GFAP), Neuron Specific Enolase (NSE), Carcinoembryonic antigen (ACE), von Willebrand factor, factor VIII related antigen (vWF), cytokeratin high molecular weight AE1-AE3, Vascular Endothelial Growth Factor (EVGF), Vascular Endothelial Growth Factor Receptor-II (EVGF-RII), Nerve growth factor receptor (NFGR), Epithelial Growth Factor (EGF), Hipoxic Inducible Factor 1 alfa (HIF-1 $\alpha$ ). DAKO Cytomation, Carpintery Ca. Santa Cruz (SC) San Ramón Ca.

Cytokeratins 7/18, vimentin, podoplanin, S-100 protein, GFAP, transthyretin and synaptophysin have been reported to be strong in choroid plexus tumors and usually are negative to CK20, EMA, CD56, CD57, CD99, NSE, and AE1/AE3 ${ }^{[5]}$.

The great majority of ependymomas display GFAP immunoreactivity, and also expressed S-100 protein, EMA, nestin neurofilament and vimentin. Focal immunoreactivity to Cytokeratins can be seen in some cases. Ki-67 labelling index of less than $4 \%$ have been associated to longer survival times ${ }^{[5]}$.

HBs stromal cells showed a variable immunoreactivity for neuroectodermal markers, such as S-100 protein, CD56, CD57, CD99, NSE, HIF- $1 \alpha$ and F-VIII but negative for GFAP and EMA ${ }^{[5]}$. Inhibin-alpha, aquaporin1 and AE1/AE3 were shown as positive markers of HBs but are not totally reliable distinguishing HBs from metastatic clear cell renal carcinoma.

This result, in conjunction with the absence of immunoreactivity for epithelial, mesenchymal, and endothelial markers, likely suggests neuroectodermal differentiation of stromal cells ${ }^{[1-3]}$. An another component, known as a cellular variant, where epithelioid tumor cells were arranged in nests encircled by capillaries and/or in pseudo rosette-like structures, was noted. GFAP immunoreactivity, which was totally absent in the epithelial cells as well as stromal cells only showing the reticular pattern, that suggesting a distinctive sign of glial differentiation in a proportion of haemangioblastoma. However, draws attention in our case the presence of papillae suggesting ependymomas with endothelial hyperplasia coming to 
form glomeruli-like structures that have not been described or ependymomas or choroid plexus Papilloma or haemangioblastoma.

Different theories of histogenesis have been considered; a vascular nature, a glial or neural, neuroectodermal, epithelial and fibrohistiocitary origin ${ }^{[1]}$. Beside its secretory and barrier functions, the choroid plexus has a novel role in attenuating the differentiation of adjacent neural progenitors ${ }^{[1]}$. It has been widely postulated neuroectodermal or neuroendocrine positivity at repeatedly Protein S-100, NSE and other neuropeptides, together with its supposed location exclusively in the central nervous system ${ }^{[4-6]}$, less defensible is the epithelial origin indicated by the occasional positivity of cytokeratins, as even the EMA negative. In other studies, the positivity for Factor XIII seems to pair it with a fibrohistiocitary origin ${ }^{[4]}$. Recently has been demonstrated in the stromal cell expression de Transretinin and transferrin, which may arise from the epithelium of choroid plexus ${ }^{[6]}$. These findings support the notion that hemangioblastoma stromal cells may originate from the embryonal plexus epithelium ${ }^{[6]}$.

It is concluded that the capillary hemangioblastoma consists of multiple cell lines endothelial cells, pericytes, stromal cells and, occasionally, haematopoietic cells all of which are neoplastic and replicate in parallel with one another. HIF- $\alpha$ is established with a concomitant induction of hypoxia-regulated genes, including EVGF, overexpression of HIF- $\alpha$ and EVGF through this signalling could explain the extraordinary capillary component of VHL-associated neoplasm. Displacement and maldifferentiation of primitive, multipotent neuroepithelial cells. The case presented may constitute a new neoplastic entity within the recently described spectrum of central nervous system tumors with ependymal features.

\section{References}

[1] Louis DN, Ohgaki H, Wiestler OD, et al. The 2007 WHO Classification of Tumours of the Central Nervous System. Acta Neuropathol (Berl). 2007; 114: 97-109. PMid: 17618441. http://dx.doi.org/10.1007/s00401-007-0243-4

[2] García-Valtuille R, Abascal F, García-Valtuille AI, et al. Adult choroid plexus papilloma of the posterior fossa mimicking a hemangioblastoma. Case report. J Neurosurg. 2000; 92: 870-2. PMid: 10794304. http://dx.doi.org/10.3171/jns.2000.92.5.0870

[3] Lowenthal A. Cerebellar hemangioblastoma, meningeal hemangioblastoma, with osseous metaplasia, hypertrophy of the choroid plexus with amorphous calcium overload, in the same subject. Acta Neurol Psychiatr (Belg). 1950; 50: 185-98. PMid: 15425346.

[4] Rickert CH, Paulus W. Tumors of the choroid plexus. Microsc Res Tech. 2001; 52: 104-11. http://dx.doi.org/10.1002/1097-0029(20010101)52:1<104::AID-JEMT12>3.0.CO;2-3

[5] Sharifi M, Ciołkowski M, Krajewski P, et al. The choroid plexus of the fourth ventricle and its arteries. Folia Morphol (Warsz). 2005; 64: 194-8.

[6] Wolburg H, Paulus W. Choroid plexus: biology and pathology. Acta Neuropathol. 2010; 119: 75-88. PMid: 20033190. http://dx.doi.org/10.1007/s00401-009-0627-8

[7] Cai C, Stephens BH, Leonard JR, et al. Posterior fossa tumor with distinct choroid plexus papilloma and ependymoma components. Clin Neuropathol. 2015 Jan 20. [Epub ahead of print] PubMed PMID: 25601059.

[8] Dulai MS, Caccamo DV, Briley AL, et al. Intramedullary papillary ependymoma with choroid plexus differentiation and cerebrospinal fluid dissemination to the brain. J Neurosurg Pediatr. 2010; 5(5): 511-7. http://dx.doi.org/10.3171/2009.12.PEDS09130

[9] Mobley B, Kalani MY, Harsh GR, et al. Papillary tumor of the spinal cord: report of 2 cases. Am J Surg Pathol. 2009; 33 (8): 1191-7. http://dx.doi.org/10.1097/PAS.0b013e31819c45ca

[10] Iwasaki T, Kato M, Horie Y, et al. A pediatric intramedullary spinal cord tumor with unusual solid-cystic and papillary features: a case report. Neuropathology. 2011 Dec; 31(6): 632-8. http://dx.doi.org/10.1111/j.1440-1789.2011.01209.x

[11] Bollo RJ, Zagzag D, Samadani U. Synchronous choroid plexus papilloma of the fourth ventricle and ependymoma of the filum terminale: case report. Neurosurgery. 2010 Nov; 67(5): E1454-9; discussion E1459. http://dx.doi.org/10.1227/NEU.0b013e3181f35144

[12] Hayashi Y, Mohri M, Nakada M, et al. Ependymoma and choroid plexus papilloma as synchronous multiple neuroepithelial tumors in the same patient: a case report and review of literature.

[13] Pasquier B, Péoc'h M, Morrison AL, et al. Chordoid glioma of the third ventricle: a report of two new cases, with further evidence supporting an ependymal differentiation, and review of the literature. Am J Surg Pathol. 2002 Oct Kopp; 26 (10): $1330-42$. 